\title{
Pour une pédagogie de la vulnérabilité
}

La proposition du pragmatisme critique

\section{Christophe Point}

\section{(2) OpenEdition Journals}

Édition électronique

URL : http://journals.openedition.org/ejpap/1961

DOI : 10.4000/ejpap.1961

ISSN : 2036-4091

Éditeur

Associazione Pragma

Référence électronique

Christophe Point, « Pour une pédagogie de la vulnérabilité », European Journal of Pragmatism and American Philosophy [En ligne], XII-7 | 2020, mis en ligne le 16 juin 2020, consulté le 26 juin 2020. URL : http://journals.openedition.org/ejpap/1961 ; DOI : https://doi.org/10.4000/ejpap.1961

Ce document a été généré automatiquement le 26 juin 2020

\section{(c) (i) $\odot$}

Author retains copyright and grants the European Journal of Pragmatism and American Philosophy right of first publication with the work simultaneously licensed under a Creative Commons AttributionNonCommercial-NoDerivatives 4.0 International License. 


\title{
Pour une pédagogie de la vulnérabilité
}

\author{
La proposition du pragmatisme critique
}

\section{Christophe Point}

1 Depuis plus d'un siècle, trois courants philosophiques; la théorie critique, le pragmatisme et le féminisme entretiennent un dialogue riche et complexe. Par l'intermédiaire de John Dewey (Laird 1988), Jane Addams (Leffers 1993), Richard Rorty (Janack 2010), Charlène Seigfried (2000), bell hooks (2003) ou encore Richard Shusterman (2003), la théorie critique et le pragmatisme ont souvent affirmé, de différentes manières, leurs volontés de participer aux réflexions et aux luttes féministes. Nous souhaitons continuer ce geste ici en étudiant son prolongement sur le terrain pédagogique. Aussi, notre problème est simple: quel est l'intérêt de ces courants philosophiques pour les pédagogues féministes?

2 L'hypothèse que nous souhaitons avancer est en revanche plus complexe: le pragmatisme peut former une base théorique intéressante pour les pédagogies féministes, si et seulement si, il apporte à ces dernières des outils conceptuels pour mieux prendre en charge et penser le "consciousness-raising": ${ }^{1}$ passage nécessaire du "gender-blindness" au "gender-consciousness" que les étudiants comme les enseignants doivent opérer pour les pratiquer. Ces deux concepts ("gender-blindness" et "gender-consciousness"), inspirés de la théorie critique, peuvent se définir comme étant l'état cognitif d'un individu qui, pour le premier concept, ignore ces biais sexistes et est aveugle à leurs effets néfastes sur autrui. Et, pour le second, l'état cognitif de celui qui a conscient de son genre, des biais que celui-ci entraîne, et, in fine, des effets de ces derniers au quotidien, dans les interactions sociales, et notamment des risques de perpétuation des dominations des hommes sur les femmes. ${ }^{2} \mathrm{Au}$ regard de la théorie critique, la possibilité, la nécessité et la difficulté d'un tel passage, pour les femmes et les hommes, sont des questions centrales pour les pédagogies féministes.

3 En effet, comment rendre responsable un individu de ses biais de genre si ce même individu ignore leurs existences et leurs conséquences sur autrui? Si on ne peut rendre responsable quelqu'un de ce qu'il ignore. Aussi il importe pour les pédagogues 
féministes d'opérer ce passage de la façon la plus efficace possible, c'est-à-dire pour le plus grand nombre d'élèves et le plus aisément possible pour l'enseignant. Or, plus l'épistémologie féministe se déploie et révèle les "privilèges" (McIntosh 1989) des hommes, et plus opérer ce passage semble pour eux difficile. En effet, pour ces derniers, ne pas reconnaitre ses privilèges devient alors une incapacité à changer son point de vue sans paniquer et est ainsi le signe d'une fragilité masculine. ${ }^{3}$ C'est pourquoi cette incapacité à "checker ses privilèges" devient la première étape en direction d'un masculinisme étroit, obtus et nuisible pour tous. Éviter cet échec à réaliser cette délicate opération de "conscientization" semble donc être un enjeu de taille pour les pédagogies féministes.

4 Face à ce problème, en quoi le pragmatisme est-il capable d'apporter des outils conceptuels pour améliorer, à la suite de la théorie critique, ce passage de l'ignorance des biais sexistes à leur conscientisation puis à la responsabilité "genrée" de l'individu envers autrui? Ce que nous souhaitons montrer ici par notre hypothèse c'est que le pragmatisme permet à la fois de révéler deux difficultés que rencontrent les pédagogies féministes, inspirées par la théorie critique, dans ce passage - ce sera le premier temps de notre argumentation et de proposer deux pistes de réponses pour répondre à ces difficultés - ce qui occupera le second temps de notre travail. Pour apporter plus de clarté au sein de notre argumentation, nous avons distingué deux "familles" de pédagogie féministe: ${ }^{4}$ l'approche critique et l'approche pragmatiste, que nous allons étudier successivement pour montrer en quoi, dans un dernier temps, ces dernières peuvent se compléter.

\section{La pédagogie critique féministe: difficultés et limites}

5 Dans un premier temps, concentrons notre propos sur les difficultés que rencontrent les pédagogies critiques féministes à réaliser cette "conscientisation genrée" (c'est-àdire le passage du "gender-blindness" au "gender-consciousness"). En effet, d'une part c'est à l'aune de ces difficultés que l'on pourra mesurer l'apport du pragmatisme en philosophie de l'éducation, ${ }^{5}$ et d'autre part, celles-ci peuvent s'identifier par deux obstacles majeurs que nous allons d'abord étudier successivement et identifier ensuite comme des limites de l'approche critique en pédagogie que le pragmatisme viendra ensuite dépasser.

\subsection{Un premier obstacle: le roi nu ne rougit plus...}

Le premier argument que nous voulons soutenir ici est le suivant: l'approche critique en pédagogie se concentre davantage sur la remise en question critique des théories éducatives que sur l'élaboration de nouveaux outils pratiques. Ce déséquilibre entre le moment du "problem-posing" et celui du "problem-solving" ralentit le processus d'émancipation auquel aspire l'approche critique (Pereira 2017: 78).

7 En effet, dans la tradition des approches critiques, matérialistes et marxistes, desquelles héritent l'Ecole de Francfort ainsi que sa déclinaison au sein du féminisme (Crenshaw 2005; Martin 1985; Kincheloe 2008), une grande rigueur est portée sur la construction du problème sur lequel va porter notre critique. Il convient d'en saisir toute la portée en maximisant en quelque sorte l'étendue de ses champs d'application, ainsi que la radicalité de sa présence au sein d'une situation. Cette approche a alors 
souvent pour conséquence de construire des concepts théoriques complexes et suffisamment généraux pour rendre compte de l'étendue et de la radicalité du problème sur lequel on travaille. Il s'en suit une sorte d'escalade à la radicalité et à l'étendue au sein des approches théoriques critiques qui tentent chacune d'appuyer leur crédibilité en compétitionnant la portée de leur critique. Cela a pour conséquence, pour la pédagogie, d'avoir des concepts extrêmement couteux théoriquement comme le capitalisme, l'aliénation, le marché, le patriarcat, etc., et qui, une fois employés, construisent une interprétation de la réalité sociale trop complexe et problématique pour les acteurs du milieu éducatif.

8 Le danger d'une telle construction théorique est alors double pour les pédagogies féministes. D'une part, l'abstraction de la réalité visée étant proportionnelle à la généralité de son interprétation théorique, le premier risque est de faire du second moment de la critique, celui de la résolution du problème, une réflexion qui sera sans cesse ajournée, reportée, et éloignée par la radicalité du problème construit. Le second risque est, par l'effort de déconstruction lui aussi proportionnel à l'étendue du problème construit, de ne pouvoir traiter théoriquement ce dernier que par une démarche argumentative basée sur l'utopie (Sandoval 2003: 202). ${ }^{6}$ Dans les deux cas, les pédagogues se retrouvent face à des problèmes qu'ils ne peuvent plus résoudre par eux-mêmes et leurs pratiques quotidiennes. Cela peut expliquer en partie pourquoi, dans certaines situations pédagogiques, certain'es féministes déclarent hésiter à se réclamer directement des théories féministes et cherchent à "contourner" les difficultés que leur filiation engendrent pour se concentrer sur des pratiques pédagogiques plus efficientes concrètement (Amboulé Abath, Campbell \& Pagé 2018: 10-1). Par exemple, si travailler avec ses élèves le caractère malsain des biais sexistes nécessite de comprendre théoriquement ce qu'est le patriarcat dans l'ensemble de sa réalité historique, économique, et politique, tout en étant attentif à ses intrications avec les discriminations sociales, raciales, culturelles, etc. Il y a fort à parier que l'éducateur ou l'éducatrice en question renonce alors à l'expliquer à ses élèves et cède au sentiment de fatalité en pensant que sa pratique est insuffisante face à un problème si complexe.

9 Ainsi, la première difficulté que la pédagogie critique apporte à certaines théories féministes, c'est de se satisfaire d'une démarche se concentrant principalement sur la construction du problème, puis de crier que le roi est nu. ${ }^{7}$ L'illusion que l'innocence déclamative de la critique suffit à transformer et performer la réalité, ici d'aller rhabiller le roi, ne peut être un atout pour la pédagogie. Même nu, si le roi continue de régner, sa honte ne durera pas longtemps. En effet, cette dernière pour être effectivement émancipatrice, a besoin d'une théorie qui construit des problèmes mais également des moyens de les résoudre (au moins partiellement) avec ses élèves. ${ }^{8}$

\subsection{Une deuxième limite: le conflit comme péché originel}

Deuxièmement, il nous semble que le pragmatisme peut s'avérer pertinent pour compléter la pédagogie critique s'il réussit à ré-orienter cette dernière hors des ornières dans laquelle le plonge une sorte "d'ontologie du conflit." Celle-ci est la seconde difficulté que nous relevons de la théorie critique: le passage du "genderblindness" au "gender-consciousness" est principalement conceptualisé par certaines théories critiques féministes à partir du prisme de la science politique (Weiler 1991: 456). Pour sortir les individus d'une "phénoménologie de la violence," l'idée est de faire 
du conflit un moyen de déplacer la lutte pour l'égalité des genres sur le terrain de la politique plutôt que sur le terrain de la violence subie individuellement ou collectivement. ${ }^{9}$ Or cette perspective est résolument conflictuelle par le fait que la reconnaissance de la domination et le combat contre cette dernière sont dans le champ politique largement pensées comme des luttes (Hill Collins 2003: 114-5). En ce sens, la présence de la figure hégélienne du maître et de l'esclave illustre bien la résolution conflictuelle de cette dialectique (Pereira 2017). Aussi certaines théories féministes pensent le problème du passage de "la prise de conscience genrée" comme une lutte pour la considération du point de vue des opprimée'es (Rancière 2004; Freire 1996; Point 2020). C'est-à-dire un processus de déconstruction de l'agnotologie des dominants qui leur donne le droit d'ignorer le point de vue que les dominés ont sur eux-mêmes et leurs réalités. ${ }^{10}$

11 Or, il nous semble que ces théories adoptent alors une conception "épistémo-politique" de la relation savoir/pouvoir comprise comme un rapport de force (au sens des théories de la domination foucaldiennes (Magar-Braeuner 2018: 162)) plutôt que comme un partage des perspectives (au sens de la standpoint theory féministe (Harding 2003)). L'approche critique met souvent en avant le politique au détriment du pédagogique: la prise de conscience genrée devient alors le lieu d'un affrontement antagoniste entre dominée's et dominante-s (Lamoureux 2010). À l'inverse, l'approche pragmatiste fait de celle-ci le lieu d'un dialogue où les perspectives dialoguent et où les acteurs peuvent s'allier pour mieux se comprendre et agir dans un intérêt mutuel (hooks 2003). En effet, loin d'ignorer la conflictualité, Dewey en fait la "gadfly of thought" (Dewey 1922: 207) dont l'importance sera déclinée sur trois niveaux de réflexions (Point 2018). Premièrement, il reconnait, comme Marx (Renault 2013), l'idée d'une lutte des classes conflictuelle au sein des sociétés humaines qui les transforme et les fait évoluer (Dewey 1930; 1935a: 61; 1935b: 259-60). Deuxièmement, il inscrit le conflit au cœur même de la logique de l'enquête, logique immanente à toutes nos pensées critiques et réflexives (Dewey 2011: 137). C'est d'ailleurs là un des traits les plus marqués de l'influence de Hegel sur la construction de la logique deweyenne (Renault 2017; Thayer 1988: 527). Enfin, au sein des luttes sociales, la compréhension de la conflictualité est pour cet auteur indispensable à la formation d'une "organized intelligence" (Dewey 1908b: 50; 1927: 326-52; 2011: 232-3) permettant de mener la lutte. C'est donc une lecture fine de ce qui est en conflit qui permet la recherche des intérêts communs à sa résolution qui est en jeu. ${ }^{11}$ Et dans ce cas, le pédagogique devient aussi important que le politique car la conflictualité s'équilibre avec la recherche de biens communs.

12 Cependant, au sein des approches critiques, le conflit devient un refus de penser la perspective d'autrui s'il n'est pas reconnu comme dominé, et permet ainsi de délégitimer le savoir situé d'autrui pour promouvoir le sien, c'est-à-dire celui des dominée's. Or, penser que la dépossession sociale produit un privilège épistémique pour les personnes subissant une domination (Hartsock 2003: 245) est un postulat qui conduit à deux dérives possibles selon Sandra Harding. Soit, cela mène à une essentialisation de la perspective des dominée's où cette dernière devient la seule perspective valable. Soit, le privilège épistémique des dominée's conduit à une subjectivation de la domination où l'individu, dans son for intérieur, devient la seule source de la perpétuation de la domination ou de l'émancipation. Dans les deux cas, cela mène à une impasse pédagogique où l'enfant se retrouve, soit sommé de choisir seul son camp dans une lutte qu'il n'a aucunement voulue (Magar-Braeuner 2018: 173), 
soit d'office désigné et réifié (Mozziconacci 2017a: 358-9) comme un individu faisant irrémédiablement parti du problème (en étant du côté des dominant·es). Bref, les pédagogies s'appuyant sur ces approches critiques nous entrainent à penser les pratiques quotidiennes à travers un prisme politique conflictuel, ce qui les rendent peu efficaces.

\subsection{Fatalité, culpabilité et désespoir. une pédagogie rabat-joie?}

Déjà en 1989, Peggy McIntosh ${ }^{12}$ s'interroge sur l'efficacité du concept de "privilège" si ce dernier ne permet pas de distinguer les avantages auxquels héritent certaines populations et qui seraient profitables d'étendre à tous et toutes et ceux qui nuisent de façon injuste aux autres populations à qui ces avantages ont été refusés. Or, réaliser cette distinction est un des enjeux et des signes de l'efficacité du pédagogue à éduquer ses élèves à la conscientisation genrée. Pourquoi une telle distinction est-elle si importante? Si les pédagogues féministes ne se préoccupent que de montrer les problèmes et les injustices que provoquent le "gender-blindness," alors les enfants se sentiront responsables de quelque chose qu'ils ignoraient auparavant, et ce d'autant plus si on subjectivise ou essentialise cette ignorance (Blidon 2012). La conscientisation genrée réalisée dans cette situation provoquera alors chez les "enfants non-privilégiés" d'une part de la colère, du ressentiment ou de la honte, et d'autre part, chez les "enfants privilégiés" de la honte, de la culpabilité ou de l'incrédulité. Ces affects négatifs, qu'ils se redoublent ou non à l'extérieur de l'espace scolaire, sont des freins conséquents au travail pédagogique (Debarbieux 1999). Dans les deux cas, le mal-être causé ne permet guère un épanouissement des enfants. ${ }^{13}$ Les études menées sur "le climat scolaire" montrent toutes que les conflits scolaires engendrés par des violences physiques ou verbales nuisent à tous les élèves, et plus particulièrement ceux qui appartiennent à des minorités discriminées (Merle 2007).

De plus, il est facile de comprendre que face à la présentation d'un problème provoquant tant d'affects négatifs lors de sa construction, sa résolution risque d'être freinée par un réflexe psychologique d'évitement du problème. La mauvaise foi, le déni, la minimisation des conséquences seront alors le lot des réactions auxquelles se confrontera l'enseignante. Or, si ces réflexes peuvent être combattus par les adultes, dont on supposera la maturité émotionnelle qui conditionne leurs responsabilités morales et citoyennes (et sur lesquelles peuvent opérer le politique), à l'inverse, si l'enfant a l'impression de ne faire que partie du problème (en étant soit bourreau soit victime), alors cet apprentissage de la conscientisation genrée lui donnera l'impression de perdre quelque chose et le mettra à mal affectivement.

Ainsi, il nous semble que ces deux difficultés (le déséquilibre du "problem-posing" par rapport au "problem-solving" et la valorisation d'une conflictualité) viennent freiner l'efficacité du travail de la pédagogie féministe, car elles développent chez l'élève des affects négatifs qui ne vont pas lui donner envie de persévérer dans ce processus éducatif, surtout pour les enfants se sentant privilégiés. En un mot, les problèmes des biais sexistes lui apparaitront si conséquents, si irrésolvables ou si "couteux" émotionnellement qu'il aura plutôt tendance à se décourager ou à nier leur existence. C'est pourquoi l'androcentrisme ${ }^{14}$ ne peut être combattu de la même façon sur le terrain de la politique comme sur celui de la pédagogie. La sensibilité et la vulnérabilité des enfants par rapport à celles des adultes imposent aux pédagogues de se munir 
d'autres outils théoriques supplémentaires pour compléter ceux construits (pour la lutte politique) par la théorie critique.

C'est pourquoi une pédagogie féministe sensible à l'importance du rôle des affects au sein du processus de cognition et de valuation complexe qu'est cette "conscientisation genrée" ne peut passer à côté du problème pédagogique de la gestion des affects lors du processus d'apprentissage (Mozziconacci 2017a: 502). Ainsi, là où une pédagogie "rabatjoie" (Ahmed 2012) répondrait aux souffrances provoquées par une domination sexiste par une exposition obstinée et sans concession de ses souffrances, nous allons voir comment une autre approche pédagogique, celle du pragmatisme, plus proche des théories du care (Seigfried 2000) (cherchant à équilibrer la production d'affects négatifs durant la phase du "problem-posing" avec d'autres affects positifs lors du "problemsolving").

\section{Les pistes de réponse de la pédagogie pragmatiste féministe}

Après avoir esquissé rapidement les difficultés auxquelles se trouvent confrontées les pédagogies critiques féministes, il nous faut tenter de trouver des pistes de réponses aux problèmes que nous avons soulevés. La valeur de la pédagogie pragmatiste, si elle veut avoir un quelconque intérêt pour les luttes féministes, se doit de répondre à ces deux questions. Premièrement, quels outils théoriques propose le pragmatisme pour ré-équilibrer la relation du "problem-posing" avec le "problem-solving" qu'amène la théorie critique? Et deuxièmement, comment remplacer la conflictualité lors du processus de conscientisation genrée par d'autres dynamiques plus efficaces pédagogiquement?

\subsection{Première piste: œuvrer à une meilleure intelligence culturelle de nos habitudes}

Pour les pédagogues pragmatistes féministes, le problème de l'équilibre du "problemposing" avec le "problem-solving" ne trouve sa résolution que dans une définition de l'éducation bien particulière. ${ }^{15}$ Celle-ci est celle de l'application intelligente de la méthode de l'enquête où le pédagogue doit se demander à chaque instant comment accompagner l'enfant à développer les outils nécessaires à la fois à la construction du problème mais aussi à sa résolution. Le pédagogue ne peut résoudre le problème à la place de l'enfant (Addams 1913: préface p. 32; Seigfried 1999: 225), ni présupposer en amont de son apprentissage la "bonne" manière de résoudre le problème (Dewey 1934: 344-5), ni encore nier l'existence du problème si l'enfant en ressent la présence. Mais surtout la tâche du pédagogue est de ne pas le laisser démuni face à celui-ci. Cela signifie, a minima, qu'il faut permettre à l'enfant de croire que ce problème est résoluble. Cet engagement, même minime, de la pédagogie pragmatiste féministe permet d'orienter la démarche pédagogique féministe dans une autre direction que celle de la pédagogie féministe uniquement critique.

19 En effet, l'épistémologie sociale pragmatiste comprend la culture comme une somme d'habitudes collectives socialement construites (Dewey 2018: 232). Ces habitudes sont des ensembles complexes de cognition, de jugement et de pratique ayant la 
particularité d'être produits par le sujet selon une économie à la fois inconsciente et consciente. La culture nous fait adopter certaines habitudes au sens où elles les désignent comme des solutions "valables" aux problèmes que rencontre le sujet. Ce dernier atteste alors inconsciemment de la validité de ces solutions car il en constate, au gré de ses expériences, leur efficacité face à ces problèmes. La collection de ces habitudes sera chez le sujet ce que l'on appelle sa culture (Cervulle 2012). Or, les effets constatés par le sujet qui orientent le choix d'une habitude par ce dernier ne sont pas des jugements objectifs mais sont, eux aussi, des constructions sociales. Notre culture nous rendra sensible à certains effets et pas à d'autres, selon une économie que l'épistémologie féministe étudie de près (McHugh 2015). Par exemple, un enseignant peut ne pas prêter attention à la répartition de la parole au sein de sa classe car en tant qu'homme il n'a jamais eu à se confronter à l'injustice de se faire couper systématiquement la parole en raison de son appartenance à un genre opprimé. Il ne développera alors aucune habitude de contrôle égalitaire de la participation des élèves dans sa classe, et ce, sans se "sentir" oppresseur envers quiconque. Il ne changera cette habitude que si, premièrement, il vit une expérience lui dévoilant les effets négatifs de celle-ci. Et deuxièmement, si une autre habitude, plus égalitaire de prise de parole entre les garçons et les filles de sa classe pour remplacer l'antécédente lui semble possible et souhaitable. En effet, nous adoptons certaines habitudes car nous en apprécions culturellement certains effets positifs, tout en ignorant les autres effets négatifs. C'est pourquoi l'épistémologie pragmatiste fait de notre intelligence des habitudes un mélange de connaissance et d'ignorance co-produite par la culture et le sujet l'adoptant.

Cette épistémologie féministe pragmatiste explique ainsi comment l'état de "genderblindness" rend l'individu ignorant des effets négatifs de ses biais sexistes et qu'il ne peut pas être déclaré seul responsable de cette ignorance. Et c'est pourquoi, sur le plan pédagogique, lorsque le ou la pédagogue mène un élève à l'état cognitif du "genderconsciousness" il est de sa responsabilité de faire en sorte que l'élève se rend compte des inconvénients d'une habitude sexiste, par une connaissance plus aigüe des effets négatifs de celle-ci, puis qu'il se mette à rechercher une autre habitude moins nuisible. Et cela n'est possible que si le sujet comprend l'intérêt de cette nouvelle habitude (qui sera alors vécu comme un changement culturel réussi) où les effets négatifs seront moins nombreux et les effets positifs identiques ou supérieures. Ainsi la conséquence de cette définition pragmatiste de l'éducation (comme construction culturelle de nouvelles habitudes) est de chercher dans la pédagogie des outils pour atteindre les finalités éducatives suivantes: 1 / faire maîtriser la méthode de l'enquête sociale, c'est-à-dire doter les élèves d'une intelligence sociale permettant une connaissance plus fine des effets sociaux de leurs habitudes. 2/ placer les élèves dans la perspective d'une éthique du care, c'est-à-dire de leur permettre de juger et de chercher à détruire les habitudes qui causent plus d'effets négatifs que positifs pour soi-même et pour autrui, sans qu'un autre se donne le droit de le faire à sa place (Paperman 2015: 13-4). 3/ faire croire en la créativité émancipatrice de chacun, c'est-à-dire d'aider les élèves à inventer de nouvelles habitudes remplaçant les anciennes, au profit de tous.

Prendre en considération ce dernier point permet de réviser le pragmatisme "classique" et de l'actualiser face aux défis de la pédagogie critique. En effet, en considérant cette épistémologie, il devient clair que l'éducation démocratique et ses écoles doivent être "des espaces où une recherche intelligente sur les normes culturelles et les modes de vie peut façonner et améliorer les habitudes" (Stitzlein 2014: 
70). L'ambition démocratique du pragmatisme implique un rôle de transformation des normes culturelles par l'éducation. Et cette transformation est un travail d'amélioration des habitudes de ces futurs citoyens (Giroux 1997). Aussi, si les habitudes sexistes sont ce que nous voulons critiquer et détruire, alors le méliorisme prônée par l'épistémologie pragmatiste (et étayé par la recherche intelligente de l'enquête et la conviction démocratique) devient une conviction "subversive et transformatrice est liée à l'action politique. [...] Les habitudes d'espoir nous font passer du statut de simples répondants réactionnaires à celui d'agents actifs" (Stitzlein 2014: 83). Ainsi, que ce soit pour bell hooks ou Patrick Shade. Donner de l'espoir en notre capacité à résoudre des problèmes aussi importants que le sexisme est en soi un travail critique dont le pédagogue ne peut se dispenser (hooks 2003; Shade 2001: 22).

\subsection{Deuxième piste: construire des outils pour améliorer le partage des perspectives}

22 Maintenant que les finalités éducatives de l'approche pragmatiste pour la pédagogie féministe ont été identifiées, reste à nous de voir si la philosophie pragmatiste a également les moyens de ces dernières. Ces moyens sont-ils des outils permettant de remplacer la conflictualité, et par là, capables d'augmenter l'efficacité pédagogique des féministes à accompagner le "consciousness-raising" de leurs élèves? Ici, nous concentrerons notre propos sur deux de ses outils: la perplexité et la "loving perception" et sur l'épistémologie pragmatiste féministe qui construit ses deux outils.

Le premier outil est conceptualisé dès 1913 par Jane Addams. ${ }^{16}$ Grâce à cette auteure le pragmatisme parviendra à se réviser et à se réformer pour répondre aux limites de la pédagogie critique. Un de ses apports majeurs a été de montrer l'importance d'une qualité épistémique jusque-là relativement méprisée, il s'agit de développer une attention à la "perplexité" des individus face aux problèmes qu'ils rencontrent. C'est en étant sensible à cette dernière que l'on peut mieux comprendre quelle est la perception de l'individu sur sa propre situation et quels sont les choix que celui-ci fait pour résoudre son problème (Addams 1913: 50, 77-8, 112-3). En effet, comme le rappellera John Dewey en 1920 en s'inspirant de Jane Addams, la perplexité est toujours la manifestation d'une situation problématique pour le sujet. Or, percevoir cette dernière donne déjà une longueur d'avance à ce sujet par rapport à un autre dans la résolution du problème.

La pensée trouve son origine dans des conflits particuliers et dans l'expérience qui occasionne perplexité et trouble. Les hommes ne pensent pas quand ils n'ont pas d'ennuis à affronter, pas de difficulté à surmonter. Une vie de confort, de succès sans effort serait une vie sans pensée et il en va de même pour une vie toute puissante et facile. Des êtres qui pensent sont des êtres qui se sentent à l'étroit et contraints dans leur vie au point de ne pouvoir conduire une action jusqu'à son terme. (Dewey 1973: 195)

Suivant cette logique, il ne s'agit plus de "plaquer" sa propre compréhension du monde sur celle de l'individu que nous voulons comprendre et aider, mais d'être d'abord sensible à sa perspective pour ensuite pouvoir l'accompagner. L'attention à la perplexité a donc ici trois avantages selon José Médina. Tout d'abord, elle permet de lutter contre la "close-mindedness" en exploitant "les avantages du conflit productif que nous devons reconnaître et valoriser la connaissance située et hétérogène de divers agents" (Medina 2012: 6). Cet usage du conflit se réalise grâce à l'ouverture d'esprit que 
l'attention à la perplexité rend possible. Puis, elle permet également de contourner la “ epistemic arrogance" qui inhibe "la capacité à s'autocorriger et à être ouvert aux corrections des autres" (Medina 2012: 31), c'est-à-dire l'humilité nécessaire à tout dialogue véritable. Et enfin, l'attention à la perplexité s'oppose à la "epistemic laziness" qui fuit toute "résistance épistémique qui est produite par la friction entre différents points de vue" (Medina 2012: 17). Cette opposition à cette forme de paresse devient une curiosité, dont John Dewey fait par ailleurs l'éloge (Dewey 1934: 180).

En pédagogie, cet outil qu'est l'attention à la perplexité permet à l'enseignant·e de se rapprocher de la perspective de l'étudiante pour mieux comprendre ce qui chez lui résiste" à la conscientisation genrée, et ainsi identifier plus vite les problèmes que celui-ci tente de résoudre et auxquels il faudra trouver, toujours avec lui, des réponses satisfaisantes pour réussir ce passage. Être attentif et ne pas vouloir résoudre tout de suite la perplexité de l'élève contribue ainsi de répondre à une des limites de la pédagogie critique repérée par Isabelle Collet (2018: 194): la tension entre la "volonté de respecter la lecture du monde des opprimées"s" (Mozziconacci 2015) d'une part, et d'autre part, la conception prédéfinie de l'enseignant·e. Cette tension existe au sein de la pédagogie critique car son épistémologie pose comme tâche aux pédagogues de "lire à travers les lignes" de l'expression des élèves une perspective prédéfinies des opprimée's (Laurin 2013: 200) qui correspond à sa théorie. Dans cette approche, la perplexité n'est alors qu'un retard, une difficulté à éliminer. Or, pour Jane Addams, au contraire, c'est vers ceux qui sont le plus "perplexe" que l'attention doit être dirigée pour mener le processus collectif de conscientisation genrée.

Toutefois, cette perplexité ne fait pas tout, et la différence de perception de celle-ci au sein d'un groupe (d'élèves ou de citoyen-ne-s (Addams 1913: 12-3)) nécessite la présence d'un deuxième outil. Celui-ci est indispensable à la pédagogie féministe selon l'approche pragmatiste car il permet de prendre en compte la réalité de la dynamique de groupe présente dans de très nombreuses situations pédagogiques. ${ }^{17}$ Cet outil, c'est la "loving perception." ${ }^{18}$ Ce concept est croisé par Nancy A. McHugh (2015: 126-9) avec le pluralisme des savoirs situés (Haraway 1988) (cherchant à comprendre la complexité de la réalité par le partage des perspectives) et s'oppose à la "arrogant perception" qui est celle de l'observateur-spectateur, hors de la situation, prétendument objectif par sa neutralité. Pour Nancy A. McHugh, cette arrogance est celle des chercheurs croyant que leurs connaissances sont supérieures à ceux qui vivent le problème en question. À l'inverse, une "loving perception" devient sensible aux transactions épistémiques entre les individus d'un groupe sur leurs différentes façons de comprendre un problème et sur leurs manières collectives de le résoudre. Elle permet d'élargir la compréhension du monde, et, en permettant aux chercheurs et aux chercheuses de se mettre à la place d'autrui, elle augmente sa capacité à dialoguer et croiser les perspectives.

En pédagogie, cet outil permet de donner une plus grande place aux affects à leurs analyses au moment même de la transmission pédagogique. Cette "loving perception," quand elle est connue et maîtrisée, permet de s'engager émotionnellement, mais aussi de se protéger des dérives d'un sur-investissement affectif (qui peut également nuire à la pratique pédagogique). Lors des stages de créativité (Huart \& Voyeux 2018: 79), de formation-accompagnement (Lafortune et al. 2018: 57-8), ou encore lors de mise en situation expérimentale pédagogique (Magar-Braeuner 2018: 166-9), les émotions que le pédagogue provoque sur ses élèves ont un double statut. Incontrôlées par les élèves ou incomprises par l'enseignant, elles sont des freins à la conscientisation genrée 
recherchée car elles deviennent motrices de conflictualités et d'opposition. Exprimées par les élèves et écoutées par l'enseignant, elles sont des données précieuses pour ajuster le processus car elles rendent possible une dynamique de partage et de coopération. C'est pourquoi, Nancy A. McHugh et d'autres pédagogues féministes, nous encouragent à affiner notre rapport aux affects que nous sollicitons et que nous percevons, en faisant de l'écoute bienveillante un outil et une vertu épistémique indispensable (McHugh 2015: 114-6).

\section{Conclusion}

Pour conclure, rappelons l'enjeu initial de notre réflexion: comprendre en quoi le pragmatisme peut fournir des outils conceptuels, complétant ceux de la théorie critique, et permettant aux pédagogues d'être plus efficaces à opérer le "consciousnessraising" dans leurs pratiques. Pour résoudre ce problème, nous avons dans un premier temps étudier l'approche critique et ces difficultés, c'est-à-dire la perte progressive d'efficacité pédagogique au fur et à mesure que la critique devient politique, abstraite et conflictuelle. Aussi les risques de subjectivation, d'essentialisation, de culpabilisation et finalement de la reproduction du déni du problème ou du fatalisme semblent importants avec cette approche, et ce de façon proportionnelle (et paradoxale) à la violence de la domination qui est combattue. Mais plus encore, c'est un certaine dérive de l'attitude critique qui semble problématique:

Cette prise de conscience ne peut s'effectuer sans une certaine douleur (hooks 1994), violence du monde social. Ce constat conduit alors à considérer comme un préalable nécessaire à une pédagogie émancipatrice la capacité à reconnaître et à supporter cette douleur, tant pour soi-même que pour les autres. (Magar-Braeuner 2018: 175)

29 Face à un tel constat, l'approche pragmatiste se distingue par la proposition d'une autre attitude venant prévenir la dérive possible de la théorie critique; une attitude où l'on cherche à développer chez soi et autrui "la pensée critique et la compassion" (McHugh 2015: 138) pour à la fois construire et résoudre les problèmes, de façon efficace et coopérative. Cela ne signifie donc ni qu'il faille "supporter" stoïquement la douleur du "consciousness-raising" pour l'enseignant·e, ni en faire un "préalable" à une pédagogie féministe. Au contraire, l'attitude pragmatiste consiste à la reconnaître pour limiter le plus possible son pouvoir de nuisance lors des pratiques pédagogiques. Pour ce faire, les épistémologues féministes et pragmatistes développent les vertus épistémiques de la perplexité, de l'humilité, de l'empathie, de la coopération, et ce dans le but de permettre aux pédagogues de construire avec elles des outils pédagogiques efficaces.

Si ces vertus (théoriques) produisent effectivement des outils (pratiques) on peut dès lors les considérer comme des convictions pédagogiques se déclinant ainsi. Premièrement, les problèmes du sexisme existent pour chacun et peuvent être résolus ensemble de façon plus efficace que chacun isolément. Deuxièmement, il est de l'intérêt de tous de résoudre ces problèmes et cela demande à tous d'être plus attentifs et responsables des effets affectifs que nos convictions provoquent chez autrui. Et enfin, troisièmement, cette responsabilité collective n'est pas qu'un poids politique ou le fardeau d'une lutte mais est également un "gain" que seule une éducation qui promeut "de manière positive, offensive et constructive la compréhension et la bienveillance essentielle à une société démocratique" (Dewey 1938: 301) rend possible. 
31 Ainsi ce travail vient s'ajouter à celui d'Irène Pereira pour promouvoir un “pragmatisme critique" (Pereira 2016). Toutefois, nous ne justifions pas l'association de la théorie critique et du pragmatisme à partir d'une perspective historique ou politique discutable (Li Vigni 2016) mais à partir de l'intérêt pédagogique de cette association, et ce, au regard du champ particulier qu'est la pédagogie féministe. In fine, nous partageons donc le point de vue Nancy A. McHugh pour qui "l'utilisation des outils de la philosophie pour créer un changement social positif et favorable à la collectivité serait une mesure du succès dans notre domaine" (McHugh 2015: 138). Il nous semble que les théories féministes peuvent s'évaluer dans le champ de la philosophie de l'éducation à l'aune des conséquences de l'usage quotidien de leurs outils respectifs auprès des élèves et des enseignant·e's. Si le pragmatisme critique permet d'opérer le "consciousnessraising" avec plus de bienveillance (c'est-à-dire une meilleure gestion des affects) et pour un plus grand nombre d'élèves (c'est-à-dire un développement plus important de la coopération des individus entre eux face à ce problème), alors dans ce cas, oui, celuici peut avoir un intérêt pour les pédagogies féministes.

\section{BIBLIOGRAPHIE}

ADDAMS Jane, (1881), “'Woman's Conscience and Social Amelioration,' The Social Application of Religion, The Merrick Lectures for 1907-8 (Cincinnati, Jennings and Graham, 1908), 41-60," in Essays: 1881-1932, vol. 12, The Major Works of Jane Addams, Charlottesville, Virginia, USA.

ADDAMS Jane, (1889), “My Friend, Julia Lathrop. Chapter IV: Early Days at Hull-House, 1889," in Essays: 1881-1932, vol. 11, The Major Works of Jane Addams, Charlottesville, Virginia, USA.

ADDAMS Jane, (1913), Democracy and Social Ethics, Citizen's Library, New York, Macmillan Co.

ADDAMS Jane, (1930), “The Second Twenty Years at Hull-House, September 1909 to September 1929, with a Record of a Growing World Consciousness," in Essays: 1881-1932, vol. 9, The Major Works of Jane Addams, Charlottesville, Virginia, USA.

AHMED Sara, (2012), “Les rabat-joie féministes (et autres sujets obstinés)," Cahiers du Genre, 53 (2), 77-98.

AMBOUlÉ ABATH Anastasie, CAMPBELl Marie-Ève \& Geneviève PAGÉ, (2018), “La pédagogie féministe: sens et mise en action pédagogique," Recherches féministes, 31 (1), 23-43.

BLIDON Marianne, (2012), “Géographie de la sexualité ou sexualité du géographe? Quelques leçons autour d'une injonction,” Annales de géographie, 687-688 (5-6), 525-42.

BREUING Mary, (2011), "Problematizing Critical Pedagogy," International Journal of Critical Pedagogy, $3(3), 2-23$.

CERVULLE Maxime, (2012), "La conscience dominante. Rapports sociaux de race et subjectivation," Cahiers du Genre, 53 (2), 37-53.

CHAPOULIE Jean-Michel, (2001), La Tradition sociologique de Chicago: 1892-1961, Paris, Seuil. 
COLLET Isabelle, (2018), “Dépasser les “éducations à’: vers une pédagogie de l'égalité en formation initiale du personnel enseignant," Recherches féministes, 31 (1), 179-97.

CRENSHAW Kimberlé Williams (2005), “Cartographies des marges: intersectionnalité, politique de l'identité et violences contre les femmes de couleur," Cahiers du Genre, 39 (2), translated by Oristelle Bonis, 51-82.

DEBARBIEUX Éric, (1999), La violence en milieu scolaire: Le désordre des choses, Paris, ESF.

DEWEY John, (1908a), "Ethics," in The Middle Works of John Dewey, 1899-1924. Volume 5: 1908, Ethics, Southern Illinois University Press, vol. 10, The Collected Works of John Dewey, 1882-1953, Charlottesville, Virginia, USA.

DEWEY John, (1908b), "Intelligence and Morals," in The Middle Works of John Dewey, 1899-1924. Volume 4: 1907-1909, Southern Illinois University Press, vol. 9, The Collected Works of John Dewey, 1882-1953, Charlottesville, Virginia, USA.

DEWEY John, (1922), "Human Nature and Conduct: An Introduction to Social Psychology," in The Middle Works of John Dewey, 1899-1924. Volume 14: 1922, Human Nature and Conduct, Southern Illinois University Press, vol. 19, Charlottesville, Virginia, USA.

DEWEY John, (1925), “Experience and Nature," in The Later Works of John Dewey, 1925-1953. Volume 1, Southern Illinois University Press, vol. 21, Charlottesville, Virginia, USA.

DEWEY John, (1927), “The Public and Its Problems. An Essay in Political Inquiry,” in The Later Works of John Dewey, 1925-1953. Volume 2: 1925-1927, Southern Illinois University Press, vol. 22, Charlottesville, Virginia, USA.

DEWEY John, (1930), “Addresses Delivered at the Dedication of the New Campus and New Buildings of the University of California at Los Angeles, 27 and 28 March 1930, Berkeley," in The Later Works of John Dewey, 1925-1953. Volume 5: 1929-1930, Southern Illinois University Press, vol. 25, Charlottesville, Virginia, USA.

DEWEY John, (1932), “Ethics (Revised Edition)," in The Later Works of John Dewey, 1925-1953. Volume 7: 1932, Ethics, Revised Edition, Southern Illinois University Press, vol. 25, Charlottesville, Virginia, USA.

DEWEY John, (1934), "Education and the Social Order," in The Later Works of John Dewey, 1925-1953. Volume 9: 1933-1934, Essays, Southern Illinois University Press, vol. 29, Charlottesville, Virginia, USA.

DEWEY John, (1935a), "Liberalism and Social Action," in The Later Works of John Dewey, 1925-1953. Volume 11: 1935-1937, Southern Illinois University Press, vol. 31, Charlottesville, Virginia, USA.

DEWEY John, (1935b), “The Future of Liberalism,” in The Later Works of John Dewey, 1925-1953. Volume 11: 1935-1937, Southern Illinois University Press, vol. 31, Charlottesville, Virginia, USA.

DEWEY John, (1938), "Democracy and Education in the World of Today," in The Later Works of John Dewey, 1925-1953. Volume 13: 1938-1939, Southern Illinois University Press, vol. 33, Charlottesville, Virginia, USA.

DEWEY John, (1973), Lectures in China, 1919-1920, Honolulu, University Press of Hawaii. DEWEY John, (2011), La formation des valeurs, translated by Alexandra Bidet, Louis Quéré \& Gérôme Truc, Paris, La Découverte.

DEWEY John, (2014), La quête de certitude: une étude de la relation entre connaissance et action, translated by Patrick Savidan, Paris, Gallimard. 
DEWEY John, (2018), Écrits politiques, translated by Jean-Pierre Cometti \& Joëlle Zask, Paris, Gallimard.

DIANGELO Robin, (2018), White Fragility: Why It's so Hard for White People to Talk about Racism, edited by Michael Eric Dyson, Boston, Beacon Press.

DORLIN Elsa, (2019), Se défendre: une philosophie de la violence, Paris, La Découverte.

FREIRE Paulo, (1996), Pedagogy of the Oppressed, new rev. ed. Penguin Books, London, Penguin Books.

GIROUX Henry A., (1997), Pedagogy and the Politics of Hope: Theory, Culture, and Schooling; a Critical Reader, Boulder, Westview Press ("The Edge: Critical Studies in Educational Theory").

HAMER CHELPI-DEN Magali, fRESIA Marion \& Éric LANOUE, (2010), “Éducation et conflits," Autrepart, 54 (2), 3-22.

HARAWAY Donna, (1988), "Situated Knowledges: The Science Question in Feminism and the Privilege of Partial Perspective," Feminist Studies, 14 (3), 575-99.

HARDING Sandra (ed.), (2003), The Feminist Standpoint Theory Reader: Intellectual and Political Controversies, 1 edition, New York, Routledge.

HARTSOCK C. M. Nancy, (2003), “Comment on Hekman's ‘Truth and Method : Feminist Standpoint Theory Revisited' : Truth or Justice ?," in Sandra Harding (ed.), The Feminist Standpoint Theory Reader: Intellectual and Political Controversies, 1st edition, New York, Routledge, 243-53.

HILL Collins Patricia, (2003), "Learning from the Outsider Within," in Sandra Harding (ed.), The Feminist Standpoint Theory Reader: Intellectual and Political Controversies, 1st edition, New York, Routledge, 103-22.

HOOKS BELL, (1994), Teaching to Transgress: Education as the Practice of Freedom, New York, Routledge. HOOKS BELL, (2003), Teaching Community: A Pedagogy of Hope, New York, Routledge.

HUART France \& Manon VOYEuX, (2018), “Quand la créativité suscite la prise de conscience féministe," Recherches féministes, 31 (1), 65-82.

JANACK Marianne (ed.), (2010), Feminist Interpretations of Richard Rorty, University Park, PA, Pennsylvania State University Press ("Re-Reading the Canon").

KINCHELOE Joe L., (2008), Knowledge and Critical Pedagogy: An Introduction, Dordrecht, Springer. LAFORTUNE Louise, GERVAIS Lise, LACHARITÉ Berthe, MAHEU Josiane, ST-CERNY Anne, GUBERMAN Nancy, COENGA-OLIVEIRA Danielle \& Priscyll ANCTIL AVOINE, (2018), "La pédagogie féministe intersectionnelle socioconstructiviste de Relais-femmes dans son travail d'accompagnement-formation: des compétences à développer," Recherches féministes, 31 (1), 45-64.

LAIRD Susan, (1988), “Women and Gender in John Dewey's Philosophy of Education," Educational Theory, 38 (1), 111-29.

LAMOUREuX Diane, (2010), Pensées Rebelles: Autour de Rosa Luxembourg, Hannah Arendt, et Françoise Collin, Montréal, Éditions du remue-ménage.

LAURIN Marie-France, (2013), "Féminisme et pragmatisme : la question de l'expérience féminine à la lumière des apports du pragmatisme à la théorie féministe," Ithaque, 13, 199-226.

LEFFERS M. Regina, (1993), "Pragmatists Jane Addams and John Dewey Inform the Ethic of Care," Hypatia, 8 (2), 64-77. 
LI VIGNI Fabrizio, (2016), "Irène Pereira, Le pragmatisme critique. Action collective et rapports sociaux," Lectures. [journals.openedition.org/lectures/20818].

MAGAR-BRAEUNER Joëlle, (2018), "La leçon de La leçon de discrimination," Recherches féministes, 31 (1), 161-78. [id.erudit.org/iderudit/1050659ar].

MARTIN Jane Roland, (1985), “Becoming Educated: A Journey of Alienation or Integration?,” Journal of Education, 167 (3), 71-84.

MCHUGH Nancy Arden, (2015), The Limits of Knowledge: Generating Pragmatist Feminist Cases for Situated Knowing, Albany, SUNY Press.

MCINTOSH Peggy, (1989), “White Privilege: Unpacking the Invisible Knapsack," Peace and Freedom Magazine (edited by Women's International League for Peace and Freedom), July-August, 10-2. MEDINA José, (2012), The Epistemology of Resistance: Gender And Racial Oppression, Epistemic Injustice, And Resistant Imaginations, 1st edition, Oxford -New York, Oxford University Press.

MERLE Pierre, (2007), "Le conflit dans l'école : question scolaire et question sociale," Le Telemaque, $31(1), 51-62$.

MOZZıConACCI Vanina, (2015), “Pédagogies Féministes et Conscientisation," Inter Pares, École doctorale EPIC, 99-103.

MOZZiConACCI Vanina, (2017a), "Le sujet du féminisme peut-il faire l'objet d'une éducation? Essai sur les théorisations féministes de la relation et de l'institution," Thèse de Philosophie, Lyon, France, Université de Lyon.

MOZZICONACCI Vanina, (2017b), “Une éducation entre personnel et politique : le "consciousness raising' dans les pédagogies féministes," in Philippe Foray \& Alan Kerian (eds.), Le métier d'enseigner: approches philosophiques, Nancy, Presses universitaires de Nancy-Éditions universitaires de Lorraine, 166-86.

PAPERMAN Patricia, (2015), “L'éthique du care et les voix différentes de l'enquête," Recherches féministes, 28 (1), 29-44. [id.erudit.org/iderudit/1030992ar].

PEREIRA Irène, (2016), Le pragmatisme critique: action collective et rapports sociaux, Paris, L'Harmattan. PEREIRA Irène, (2017), Paolo Freire, pédagogue des oppriméee's: une introduction aux pédagogies critiques, N'autre Ecole, 10, Libertalia. [editionslibertalia.com/catalogue/nautre-ecole/10-paulo-freirepedagogue-des-opprime-e-s].

POINT Christophe, (2017), “Quelle valeur a notre enseignement aux yeux des élèves?

Prolongement de la théorie de la valuation de Dewey dans la réflexion pédagogique," Les ateliers de l'éthique / The Ethics Forum, 12 (1), 4-20.

POINT Christophe, (2018), "What's the Use of Conflict in Dewey? Toward a Pedagogy of Compromise," Education and Culture: The Journal of the John Dewey Society, 34 (2), 69-88.

POINT Christophe, (2020), “Chapitre 2. Paulo Freire \& John Dewey: d'un dialogue impossible à un conflit constructif ?," in Nassira Hedjerassi (ed.), Les pédagogies émancipatrices: Actualités et Débats, présenté par Irène Pereira, Vulaines sur Seine, Édition Du Croquant.

RANCIÈRE Jacques, (2004), Le maître ignorant, Paris, 10/18.

RENAULT Emmanuel, (2013), "Dewey, Hook et Mao : quelques affinités entre marxisme et pragmatisme/Dewey, Hook, Mao: On Some Affinities between Marxism and Pragmatism," Actuel Marx, 54, 138-57. 
RENAULT Emmanuel, (2017), "Dewey et la Science de la logique," in Gilbert Gérard \& Bernard Mabille (eds.), La "Science de la logique" au miroir de l'identité ("Bibliothèque philosophique de Louvain 98"), Louvain Paris Bristol, CT, Peeters Publishers.

SANDOVAL Chela, (2003), "U.S. Third World Feminism," in Sandra Harding (ed.), The Feminist Standpoint Theory Reader: Intellectual and Political Controversies, New York, Routledge, 195-212. SEIGFRIED Charlene Haddock, (1999), "Socializing Democracy: 'Jane Addams and John Dewey'," Philosophy of the Social Sciences, 29 (2).

SEIGFRIED Charlene Haddock, (2000), "Feminist Ethics and the Sociality of Dewey's Moral Theory," Transactions of the Charles S. Peirce Society, 36 (4), 529-34.

SHADE Patrick, (2001), Habits of Hope: A Pragmatic Theory, Nashville, Vanderbilt University Press. SHUSTERMAN Richard, (2003), "Somaesthetics and The Second Sex: A Pragmatist Reading of a Feminist Classic," Hypatia, 18 (4), 106-36.

STITZLEIN Sarah M., (2014), "Habits of Democracy: A Deweyan Approach to Citizenship Education in America Today," Education and Culture, 30 (2), 61-86.

THAYER Horace Standish, (1988), "Review of Review of Logic: The Theory of Inquiry John Dewey, the Later Works, 1925-1953, Vol. 12, by Jo Ann Boydston and Ernest Nagel," Transactions of the Charles S. Peirce Society, 24 (4), 521-39.

WEILER Kathleen, (1991), “Freire and a Feminist Pedagogy of Difference," Harvard Educational Review, 61 (4), 449-75.

\section{NOTES}

1. Ce concept que l'on peut traduire (assez maladroitement) par "éveil des consciences," ou "conscientisation genrée," est développé, en partie par les travaux de Vanina Mozziconacci (Mozziconacci 2017b), mais provient à l'origine des théories critiques féministes (Weiler 1991).

2. On peut également comprendre ce passage en le comparant avec le processus que les pédagogues anti-racistes tentent d'effectuer pour mener leurs élèves du "color-blindness" au "color-consciousness" (Cervulle 2012).

3. Là aussi cette fragilité est similaire à bien des égards à la "white fragility" (DiAngelo 2018).

4. Cette distinction théorique n'est pas à l'abri des remises en question sur le plan historique ou de la pratique, mais par elle nous voulons surtout mettre en lumière deux "attitudes" pédagogiques distinctes pour les discuter. La raison en est qu'à nos yeux, l'une des premières tâches de la philosophie de l'éducation pour la pratique pédagogique est d'apporter aux pédagogues un regard clair sur leurs "attitudes" professionnelles pour leur permettre de faire des choix lucides à partir de ces dernières.

5. Il faut cependant noter que ses deux traditions philosophiques, la théorie critique et le pragmatisme, ont largement échangé entre elles et se sont mutuellement influencées, et ce malgré certains désaccords théoriques importants. Il existe donc plusieurs courants au sein de chacun de ses approches que nous ne pouvons distinguer ici dans cette argumentation schématique par nécessité, cependant on se reportera au travail de Mary Breuing proposant une revue de littérature à ce sujet (Breuing 2011).

6. Malgré cette critique classique de l'utopie déréalisante et démobilisatrice, une approche féministe et pragmatiste, à la suite des travaux de Richard Rorty (Janack 2010) et stimulée par les travaux de chercheure's comme Vanina Mozzicanoci (Mozziconacci 2015: 6) est cependant possible et consiste une piste intéressante. 
7. Nous faisons référence ici au conte de Andersen, publié en 1837, intitulé Les Habits Neufs de l'empereur.

8. Prévenons un possible malentendu. On notera que cette première difficulté, où la pratique pédagogique reproche à la théorie de construire des problèmes trop difficiles à résoudre, n'est pas propre aux théories féministes. Au contraire, il ne s'étend qu'à certaines approches inspirées par la théorie critique qui font abstraction de la pratique et oublient que l'application quotidienne pédagogique ne peut s'intéresser qu'à des problèmes auxquels elle peut contribuer à résoudre, et non à des problèmes lui paraissant irrésolvables. De plus, par cette première difficulté soulevée, nous n'insinuons ni que la pédagogie féministe peut se passer de théorie féministe, ni que les problèmes liés aux biais sexistes ne sont pas complexes et ne dépassent pas largement le champ pédagogique.

9. "Quand les proies se mettent à chasser, elles ne deviennent pas chasseurs à leur tour. Elles se défendent par nécessité. Toutefois, dans la généralisation d'un tel monde de prédation, c'est à la transformation de tou'te's en proies que l'on assiste. C'est l'éradication de toute altérité ou, plutôt, le rabattement du possible dans l'ordre de la menace et du danger; c'est aussi l'éradication de toute conflictualité politique" (Dorlin 2019: 209).

10. “Agnotologie [...] le concept d'ignorance permet de saisir l'asymétrie entre l'exploitation cognitive et gnoséologique des dominée's et les infinis bénéfices sociaux et symboliques dégagés

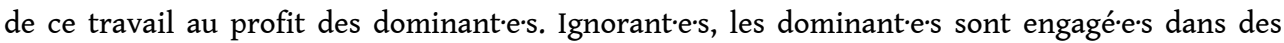
postures cognitives qui leur épargnent à proprement parler de 'voir' les autres, de s'en soucier, de les prendre en compte, de les connaitre, de les considérer" (Dorlin 2019: 207).

11. "To claim that intelligence is a better method than its alternatives, authority, imitation, caprice and ignorance, prejudice and passion, is hardly an excessive claim" (Dewey 1925: 394).

12. "For this reason, the word 'privilege' now seems to me misleading. We want, then, to distinguish between earned strength and unearned power conferred systematically. [...] But not all of the privileges on my list are inevitably damaging. [...] Others, like the privilege to ignore less powerful people, distort the humanity of the holders as well as the ignored groups. We might at least start by distinguishing between positive advantages which we can work to spread, and negative types of advantages which unless rejected will always reinforce our present hierarchies" (McIntosh 1989: 3).

13. Particulièrement au sein des lieux d'éducation situés sur des zones de conflits armés, l'étude des impacts des affects négatifs liés à des injustices ou des discriminations sur l'apprentissage des enfants sont sans appel (Hamer, Fresia \& Lanoue 2010).

14. Celui-ci est ici entendu comme le mouvement inverse de la "conscientisation genrée" promu par le féminisme.

15. "Une telle philosophie [pragmatiste] verrait s'ouvrir devant elle un vaste champ critique. Mais son esprit critique serait tourné contre la domination exercée par les préjugés, les intérêts étroits, les coutumes routinières et l'autorité provenant d'institutions séparées des fins humaines au service desquelles elle se place. Cette tâche négative ne serait que le revers du travail créatif de l'imagination lorsque cette dernière désigne de nouvelles possibilités que la connaissance de l'effectif dévoile et lorsqu'elle projette des méthodes pour leur réalisation dans la modeste expérience quotidienne du genre humain" (Dewey 2014: 326).

16. Cette pédagogue féministe pragmatiste a travaillé au carrefour de la pédagogie, de l'action sociale et de la sociologie, à Chicago au début du siècle dernier. Et, en plus d'être la première femme américaine à recevoir le Prix Nobel de la paix en 1931, elle a eu une influence conséquente d'une part en philosophie de l'éducation (en discutant et inspirant John Dewey par exemple (Seigfried 1999: 225)) et en sociologie urbaine (en inspirant de nombreuses méthodes qui feront l'originalité de la sociologie interactionniste de la première école de Chicago (Chapoulie 2001: 23-50)). 
17. En effet, dans la majorité des systèmes d'éducation occidentale, le professeur n'est plus le précepteur d'un seul enfant mais est face à un groupe d'élèves, au sein d'un groupe de niveau ou d'une classe.

18. Pour le pragmatisme, les affects prennent part aux valeurs morales (Point 2017) et c'est pourquoi l'empathie "fournit le point de vue 'intellectuel' le plus performant" en tant que "outil, 'par excellence,' pour résoudre les situations complexes” selon John Dewey (1932: 270) et John Stuart Mill (Dewey 1908a: 267-9). Cependant, c'est Jane Addams qui en fera un outil pédagogique en soi avec son concept épistémique de la "sympathetic understanding" (1881: 51; 1889: 50; 1930: 408).

\section{RÉSUMÉS}

Le féminisme est un mouvement théorique et politique pluriel et en perpétuelle évolution. Aussi ce travail se situe dans les champs de la philosophie de l'éducation et plus particulièrement au sein des débats entre plusieurs courants philosophiques se réclamant du féminisme. Il cherche à évaluer l'intérêt de deux de ces approches philosophiques au regard de la construction d'une pédagogie féministe. Se trouve ainsi comparées l'approche de la théorie critique (proche du marxisme matérialiste et de l'école de Francfort) et l'approche pragmatiste (issue des travaux de Jane Addams et John Dewey) par rapport au problème du "consciousness-raising" en pédagogie. Ce dernier présente en effet des difficultés à l'approche critique qui seront exposées dans la première partie de l'argumentation. L'hypothèse de notre travail est que le pragmatisme peut apporter des outils théoriques répondant à ses difficultés et venant ainsi compléter ceux de la théorie critique au profit de la pédagogie féministe. Ainsi la deuxième partie de notre argumentation se concentre sur l'intérêt épistémique de ces outils pédagogiques que propose le pragmatisme. Ces derniers sont rendus possibles par la valorisation épistémique de la perplexité et de l'empathie, et nous verrons en quoi ils permettent d'augmenter l'efficacité du "consciousness-raising" lors des pratiques pédagogiques quotidiennes.

\section{AUTEUR}

\section{CHRISTOPHE POINT}

doctorant en philosophie de l'éducation, cotutelle à l'Université Laval (IDEA) Canada et Université de Lorraine (LISEC) France

chris_point[at]hotmail.fr 